\title{
Actualización sobre mecanismos de tumorogénesis hipofisaria
}

\author{
Update on mechanisms of pituitary tumorigenesis
}

\section{Atualização sobre mecanismos de tumorigênese hipofisária}

\section{Laura Anahi Cecenarro1, Gabriela Deisi Moyano Crespo², Jorge Humberto Mukdsi3}

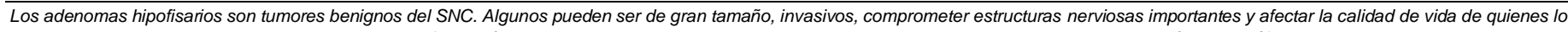

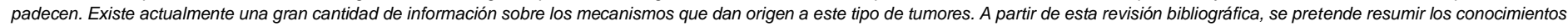
actualizados sobre la enfermedad y sus causas.

Conceptos clave:

¿Qué se sabe sobre el tema?

- Los adenomas hipofisarios son tumores benignos en la mayoría de los casos.

- El conocimiento de la biología de los tumores hipofisarios incrementó notablemente en los últimos años.

¿Qué aporta este trabajo?

- Este trabajo aporta conocimientos actualizados sobre los mecanismos que contribuyen a la tumorogénesis hipofisaria.

1- Médica Cirujana. Especialista en Endocrinología. Docente Universitaria- Universidad Nacional de Córdoba. Centro de Microscopía Electrónica. Facultad de Ciencias Médicas. INICSA.CONICET-UNC. Hospital Privado Universitario de Córdoba, Córdoba, Argentina. E-mail de contacto: laurycece@hotmail.com

2.- Médica Cirujana. Doctora en Medicina y Cirugía. Especialista en Patología. Docente UniversitariaUniversidad Nacional de Córdoba. Centro de Microscopía Electrónica. Facultad de Ciencias Médicas. INICSA.CONICET-UNC. Córdoba, Argentina. 3.- Médico Cirujano. Doctor en Medicina y Cirugía. Magíster en Bioética. Especialista en Patología. Docente Universitario- Universidad Nacional de Córdoba. Centro de Microscopía Electrónica. Facultad de Ciencias Médicas. INICSA.CONICET-UNC. Córdoba, Argentina.

Recibido: 2020-06-21 Aceptado: 2021-11-27

DOI: http://dx.doi.org/10.31053/1853.0605.v78.n4.29189 (c) (1) (8)

(C) Universidad Nacional de Córdoba

\section{Resumen:}

Los adenomas hipofisarios son neoplasias intracraneales que surgen de las células del lóbulo anterior de la glándula, benignos y de crecimiento lento en su gran mayoría. Sin embargo, un pequeño porcentaje puede mostrar un comportamiento clínicamente agresivo y diseminarse localmente y/o a distancia como verdaderas neoplasias malignas. Durante los últimos años, se observó un importante avance en el conocimiento de la biología de los tumores hipofisarios, identificándose mutaciones de las líneas germinal, somática y mecanismos epigenéticos. Objetivo: revisar la bibliografía actualizada sobre los mecanismos que contribuyen a la tumorogénesis hipofisaria. Fuente de datos: se realizó una búsqueda bibliográfica utilizando las bases de datos de MEDLINE (PubMed), LILACS y Google Scholardesde 2010 hasta abril de 2020. Conclusión: El conocimiento e información sobre los mecanismos asociados a la formación de tumores hipofisarios incrementó a lo largo de las últimas décadas, y se reconocen nuevas vías de desarrollo neoplásico. Sin embargo, en la actualidad existen pocos enfoques terapéuticos para actuar específicamente sobre la vía de génesis tumoral subyacente identificada en cada caso.

Palabras claves: hipófisis; adenoma; carcinogénesis

Abstract:

Pituitary adenomas are intracranial neoplasms that originate from the adeno-pituitary cells, are mostly benign and slow growing. However, a small percentage can be aggressive and spread locally and / or remotely as malignancies. In recent years, progress has been made in understanding the biology of pituitary tumors, identifying mutations in the germline, somatic lines, and epigenetic mechanisms. Objective: review the updated bibliography on the mechanisms of pituitary tumorigenesis. Data source: bibliographic search was performed using the MEDLINE (PubMed), LILACS and Google Scholar databases since 2010 to April 2020. Conclusion: Knowledge and information on pituitary tumorogenesis mechanisms increased in recent decades, and new neoplastic pathways are recognized. However, there are currently few therapeutic approaches to act specifically on the underlying tumor genesis pathway identified in each case.

Keywords: pituitary; adenoma; carcinogenesis

\section{Resumo}

Os adenomas da hipófisesão neoplasias intracranianas que surgem das células do lobo anterior da glândula, principalmente benignas e de crescimento lento. No entanto, uma pequeña porcentagem pode mostrar um comportamento clínicamente agressivo e se espalhar local e / ou remotamente como verdadeiras malignidades. Nos últimos anos, foi observado um avanço importante no conhecimento da biologia dos tumores hipofisários, identificando mutações na linha germinativa, linhas somáticas e mecanismos epigenéticos. Objetivo: revisar a bibliografía atualizada sobre os mecanismos que contribuem para a tumorigênese hipofisária. Fonte de dados: foi realizada uma pesquisa bibliográfica nas bases de dados MEDLINE (PubMed), LILACS e Google Scholar de 2010 a abril de 2020. Conclusão: O conhecimento e as informações sobre os mecanismos associados à formação de tumores hipofisários aumentaram à medida que nas últimas décadas, e novos caminhos de desenvolvimento neoplásico são reconhecidos. No en tanto, existem atualmente poucas abordagens terapêuticas para atuar específicamente na via de gênese do tumor subjacente identificada em cada caso. 


\section{INTRODUCCIÓN}

La glándula hipófisis es la principal reguladora de la homeostasis endócrina mediante la secreción de hormonas tróficas. Se compone de la adenohipófisis (lóbulo anterior y lóbulo intermedio) y la neurohipófisis (lóbulo posterior). Regula el crecimiento, metabolismo, reproducción, lactancia y la respuesta al estrés. Si existe alguna alteración en este sistema, aparece la desregulación hormonal y la expansión celular causando diversas morbilidades, calidad de vida deteriorada y aumento de la mortalidad ${ }^{1}$. El lóbulo anterior presenta cinco tipos de células que secretan las hormonas: tirotrofina (TSH); adrenocorticotrofina (ACTH); somatotrofina $(\mathrm{GH})$; prolactina (PRL); foliculoestimulante $(\mathrm{FSH})$ y luteinizante $(\mathrm{LH})$. Las células del lóbulo intermedio secretan proopiomelanocortina, un precursor de la hormona estimulante de los melanocitos, ACTH y endorfinas. El lóbulo posterior secreta oxitocina y arginina vasopresina. La adenohipófisis también contiene células endoteliales, pericitos y células foliculo-estrelladas no endocrinas, que producen factores de crecimiento y citocinas regulando la homeostasis pituitaria y favoreciendo la maduración de las células madre hipofisarias ${ }^{2}$. Ésta última población de células, muy estudiada en la actualidad, participa en los mecanismos de iniciación, progresión, recurrencia y resistencia al tratamiento de los tumores hipofisarios ${ }^{3}$. Estas lesiones, son neoplasias intracraneales, de crecimiento lento, con una prevalencia estimada de $16,7 \%$ (14,4\% en autopsias y $22,5 \%$ en estudios radiológicos) $)^{4}$. Generalmente no metastatizan, por lo tanto, se los conoce como "adenomas", sin embargo, un pequeño porcentaje puede mostrar un comportamiento clínicamente agresivo y diseminarse localmente $\mathrm{y} / \mathrm{o}$ a distancia como verdaderas neoplasias malignas. Recientemente se ha propuesto el término "tumor neuroendocrino pituitario" o "PitNET" para este tipo de lesiones ${ }^{5}$ y se clasifican clínicamente por sus características de secreción excesiva de hormonas o por síntomas de compresión de estructuras vecinas. La caracterización histológica se ha basado en la tinción inmunohistoquímica de las hormonas pituitarias y la reciente incorporación de factores de transcripción (PIT1 para los linajes GH, prolactina y TSH; SF1 para los linajes de gonadotrofinas y TPIT para el linaje de $\mathrm{ACTH}$ ). Por lo tanto, el diagnóstico final se basa en la combinación del cuadro clínico y la evaluación histológica (inmunomarcación de hormonas y factores de transcripción) ${ }^{6}$.

Durante los últimos años, se observó un importante avance en el conocimiento de la biología de los tumores hipofisarios, identificándose mutaciones de las líneas germinal, somática y mecanismos epigenéticos. En esta revisión, pretendemos realizar una actualización de los mecanismos que contribuyen a la tumorogénesis hipofisaria y orientar el análisis a la búsqueda de mejores decisiones preventivas y terapéuticas.

\section{MATERIALES Y MÉTODOS}

Para la elaboración de este artículo de revisión, se llevó a cabo una búsqueda bibliográfica utilizando las bases de datos de MEDLINE (PubMed), LILACS y Google Scholar. Las mismas se realizaron desde 2010 hasta abril de 2020. Se incluyeron artículos científicos en idioma español e inglés. Adicionalmente se realizaron búsquedas bibliográficas a partir de las citas mencionadas en publicaciones científicas selectas referidas a la temática. Las palabras clave utilizadas para la búsqueda fueron "hipófisis", "adenoma" y "carcinogénesis", como así también, los apellidos de los principales autores referentes. Estos términos se fueron combinando y traduciendo al inglés para la búsqueda en las distintas bases de datos. Los artículos encontrados fueron revisados por todos los autores, se analizó su contenido y se excluyeron aquellos que no se correspondían con la temática y/o objetivo del presente trabajo.

\section{RESULTADOS}

La fisiopatología de los adenomas hipofisarios es compleja y varía entre los diferentes tipos de tumores. Mediante el análisis de la inactivación del cromosoma $X$, se ha demostrado que estas neoplasias, resultan en su mayoría de la expansión clonal de células adenohipofisarias ${ }^{1}$, mediante la participación de eventos genéticos y epigenéticos, estimulación hormonal, sobreproducción de factores de crecimiento, desregulación de miARNs y participación de células madres, que promoverían el desarrollo y crecimiento tumoral ${ }^{7}$ (Figura 1).

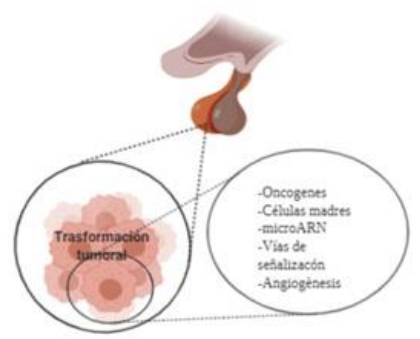

Figura 1: Esquema de la actual hipótesis de la patogenia de los adenomas hipofisarios. Se destaca el interjuego de múltiples mecanismos que participarían del origen tumoral. Diseño realizado en BioRender.com

\section{Ciclo celular y su regulación}

La división celular consta de 4 fases: fase $S$ (síntesis del ADN), fase $M$ (mitosis) y 2 fases de crecimiento y transición, llamadas fases $G$ (del inglés gap: intervalo). Las quinasas dependientes de ciclinas (CDK) controlan la progresión de las células a través de las distintas fases del ciclo celular. La actividad de la CDK está modulada por sus activadores (ciclinas) o inhibidores $(\mathrm{CDKI})^{8}$. La progresión del ciclo celular tiene reguladores negativos, específicamente las familias de inhibidores CDK INK4 y Cip/Kip ${ }^{8,9}$. La proteína supresora de tumores $\mathrm{Rb}$ también regula negativamente la entrada en el ciclo celular y la progresión $\mathrm{G} 1 / \mathrm{S}$, mientras que PTTG, factor que fuera descripto en adenohipófisis, 
participa tanto en G1/S y como en la inestabilidad cromosómica ${ }^{10}$. Diversos modelos experimentales han sido utilizados para la comprensión de la participación de las proteínas reguladoras del ciclo celular en el desarrollo de adenomas hipofisarios. Ratones knockout para inhibidores de CDK, incluyendo p18 y p27, desarrollan tumores pituitarios $^{9}$, mientras que los modelos knockout para p21/Rb, p27/Rb ${ }^{11}$, p16/p18 ${ }^{12}$, p27/ciclina $E^{13}, p 27 / p 18, p 21 / p 18^{14}$, y CDK4/p2 $7^{15}$, exhiben un menor período de latencia para la formación de tumores o incluso un incremento del tamaño de los adenomas hipofisarios. En tumores pituitarios humanos se han demostrado mutaciones de la línea germinal, así como subexpresión o metilación del ADN de los inhibidores de $\mathrm{CDK}^{16}$.

\section{A) Mecanismos Genéticos \\ 1. MUTACIONES DE LA LÍNEA GERMINAL}

Los tumores hipofisarios asociados con mutaciones en la línea germinal pueden presentarse como parte de una enfermedad aislada o sindrómica.

\subsection{Adenoma hipofisario familiar aislado (FIPA)}

Dentro de este grupo se identificaron alteraciones en diferentes genes: el de la proteína de interacción del receptor de aril-hidrocarburo (AIP) que representa el 10-20\% de FIPA, el de GPR101 en el acrogigantismo ligado al cromosoma X (XLAG) (aunque se identificó principalmente como mutación de novo, también se ha descrito en familias) y el grupo restante aún sin causa genética conocida ${ }^{17}$.

- AIP (Supresor Tumoral): esta proteína interactúa con el receptor de hidrocarburos de arilo (AHR), proteínas de choque térmico y otras proteínas, cumpliendo un rol importante en la modulación del AMPc y las vías de señalización celular ${ }^{17}$. Los FIPA por mutación AIP se transmiten por herencia autosómica dominante y presentan penetrancia incompleta. Los pacientes tienen un fenotipo clínico distinto: muestran un inicio más temprano de la enfermedad con adenomas de mayor tamaño y son predominantemente secretores de $\mathrm{GH}$ escasamente granulados, localmente invasivos y pueden desarrollar apoplejía ${ }^{18}$. Así también es menos probable que estos tumores respondan a los análogos de somatostatina (SSA) de primera generación ${ }^{19}$. Las recomendaciones actuales sugieren la detección de mutaciones AIP en pacientes sin características sindrómicas y cualquiera de los siguientes criterios: tumores hipofisarios de inicio en la infancia, tumores hipofisarios familiares, o macroadenomas a la edad de 30 años o menos ${ }^{20}$.

- Acrogigantismo ligado al X (XLAG): es producido por microduplicaciones en el cromosoma Xq26.3 que amplifican el gen GPR101, el cual codifica para un receptor huérfano acoplado a proteínas G. GPR101 se acoplaría a la proteína estimuladora de $G \alpha$, que activa la adenil ciclasa y aumenta la producción de $\mathrm{AMPc}^{21}$. Los pacientes presentan una marcada hipersecreción de GH yPRL en más del $85 \%$ de los casos a causa de adenoma o hiperplasia hipofisaria ${ }^{22}$, ocasionando aceleración de la velocidad de crecimiento desde muy temprana edad. La enfermedad se produce generalmente por una mutación de novo que se manifiesta como enfermedad de la línea germinal o mosaico, pero también se han de descripto algunos casos familiares. La penetrancia total observada en XLAG familiar puede llegar a ser del $100 \%$, por lo que vale la pena considerar el diagnóstico preimplantacional o el cribado prenatal en las madres afectadas y en los fetos femeninos de los hombres afectados (aunque todavía no se ha demostrado la transmisión de hombre a mujer ${ }^{22}$.

- Nuevas variantes de la línea germinal: la gran mayoría de FIPA no tiene una base genética establecida: aproximadamente el $85 \%$ de la cohorte FIPA analizada por Daly y col. fueron negativos para mutaciones AIP $^{18}$. Se han descrito tanto familias homogéneas (familias que presentan el mismo tipo de adenoma en todos los individuos afectados) como heterogéneas. El patrón de herencia es autosómico dominante con baja penetrancia ${ }^{17}$. En la Figura 2 se identifican otras variantes de línea germinal, que pueden predisponer a tumores familiares, pero ninguno de los datos publicados respalda de manera convincente la presencia establecida de un gen adicional que causa FIPA.

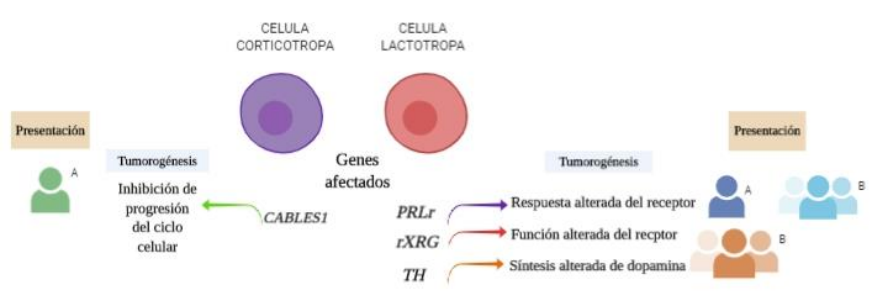

Figura 2: Algunas variantes de línea germinal, que pueden predisponer a tumores familiares. Diseño realizado en BioRender.com

\subsection{Adenomas hipofisarios en el contexto de síndromes familiares}

- MEN1 (Gen Supresor Tumoral): se encuentra en el cromosoma $11 q 13$ y codifica la proteína menina, que actúa en la regulación de la transcripción, la estabilidad del genoma y la proliferación celular ${ }^{1}$. Basado en el hecho de que se observa pérdida de heterocigosidad $(\mathrm{LOH})$ en la línea germinal que afecta al cromosoma 11q13 esta LOH se considera un golpe inicial, seguido de la mutación somática MEN1 como segundo golpe, que conduce a la formación de tumores ${ }^{26}$. Los tumores hipofisarios con mutación MEN1 tienden a ser más grandes e invasivos y están asociados a adenomas paratiroideos y pancreáticos en el mismo paciente.

Recientemente, se ha descripto en algunas familias un cuadro clínico similar a MEN1, ahora denominado MEN4, asociado a la mutación de los inhibidores de la quinasa dependientes de ciclina, principalmente p27 (CDKN1B) y raramente en p21 (CDKN1A), p15 (CDKN2B) y p18 $(\mathrm{CDKN} 2 \mathrm{C})^{27}$. Estos pacientes se caracterizan por presentar adenomas hipofisarios y de las glándulas paratiroideas, angiomiolipoma renal, tumor suprarrenal no funcionante, fibromas uterinos, gastrinoma, tumor carcinoide bronquial, carcinoma papilar de tiroides y carcinoma gástrico. El tipo de tumor hipofisario más frecuente es el somatotropinoma.

- Complejo de Carney (CNC): alrededor del 60\% de los casos del CNC son causados por la mutación de PRKAR1A (subunidad reguladora de 
la proteína quinasa tipo I-alfa dependiente de $\mathrm{AMPC}$ ), un componente clave de la vía de señalización de AMPc. Esta mutación, se encuentra implicada en la tumorogénesis endocrina ya que conduciría a un codón de parada prematuro y a la alteración del ARNm, provocando una mayor actividad de PKA. Hasta el $75 \%$ de los pacientes exhiben hipersecreción de PRL y GH ${ }^{28}$.

- Blastoma hipofisario: es un tumor embrionario de la hipófisis, de presentación poco frecuente, potencialmente mortal, que aparece en la primera infancia y frecuentemente causa enfermedad de Cushing. Se incluye dentro del síndrome DICER1, el cual está asociado a una mutación heterocigota en la línea germinal del gen DICER1, que codifica para endorribonucleasa, importante en el procesamiento de microARN $^{29}$.

- Feocromocitoma/paraganglioma y adenoma hipofisario: si bien juntos no forman parte de ningún síndrome tumoral endocrino, en raras ocasiones pueden aparecer en el mismo paciente o familia. La coexistencia de las dos enfermedades puede deberse a una coincidencia, pero también a un mismo mecanismo patogénico. Cada vez hay más casos descriptos con esta combinación de enfermedades, especialmente por mutación de los genes de succinato deshidrogenasa $(\mathrm{SDHx})^{30}$.

- Otras mutaciones de la línea germinal: se han identificado mutaciones en MLH1 y MSH2 en pacientes con tumores secretores de ACTH de crecimiento agresivo ${ }^{31}$. En USP8, comúnmente estudiadas como mutaciones somáticas en los adenomas corticotróficos, ahora también descriptas en un niño con características dismórficas, retraso en el desarrollo y tumor corticotropo ${ }^{32}$. En algunas publicaciones, se han informado tumores hipofisarios en pacientes con esclerosis tuberosa. Actualmente no está claro si estos están realmente relacionados con las mutaciones TSC1 o TSC2 o son hallazgos coincidentes ${ }^{33}$.

\section{MUTACIONES SOMÁTICAS}

- GNAS: codifica la subunidad a estimulante de la proteína G y muestra las mutaciones somáticas más frecuentes de adenomas somatotropos, confirmadas más recientemente a través del genoma completo $(W G S)^{34}$ y la secuenciación del exoma completo (WES $)^{35}$. Las mutaciones afectan el codón 201 o 227, alterando la actividad GTPasa de la proteína y conduciendo a una actividad prolongada de adenilato ciclasa con aumento en los niveles de AMPc, lo que impulsa la tumorogénesis. Estos tumores suelen ser más pequeños, menos invasivos y con mejor respuesta a los SSA ${ }^{36}$. La expresión del receptor 2 de dopamina también se encuentra aumentada en estos tumores, lo que predice mejor respuesta a los agonistas de dopamina ${ }^{37}$. Los pacientes con mosaicismo somático para el codón 201 GNAS desarrollan el síndrome de McCune-Albright, que se caracteriza por hiperplasia/tumor somatotropo o mamosomatotropo, displasia fibrosa poliostótica, manchas de café con leche y pubertad precoz ${ }^{38}$.

- USP8: mutación somática en la peptidasa específica de ubiquitina 8 (USP8), presente en los adenomas hipofisarios (35-62\% de los adenomas corticotropos esporádicos). USP8 es una desubiquitinasa, que pertenece a la familia de las peptidasas específicas de ubiquitina.
La ubiquitinación es una modificación postraduccional reversible que regula la estabilidad de diversas proteínas. USP8 elimina la parte de ubiquitina e impide así la degradación. Una de sus dianas en las células corticotropas es la regulación negativa del receptor de factor de crecimiento epidérmico (EFGR). Así, las mutaciones de USP8 producen una actividad elevada de desubiquitación y una señalización constante de EGFR que tiene como consecuencia la hiperproducción de $\mathrm{ACTH}^{39}$.

El análisis de los datos de secuenciación del exoma completo y el genoma completo de los tumores pituitarios evidenció un bajo número de mutaciones somáticas en todos los subtipos ${ }^{34,35}$. Pocos genes muestran mutaciones recurrentes, y los mismos se mencionan en la Figura 3.

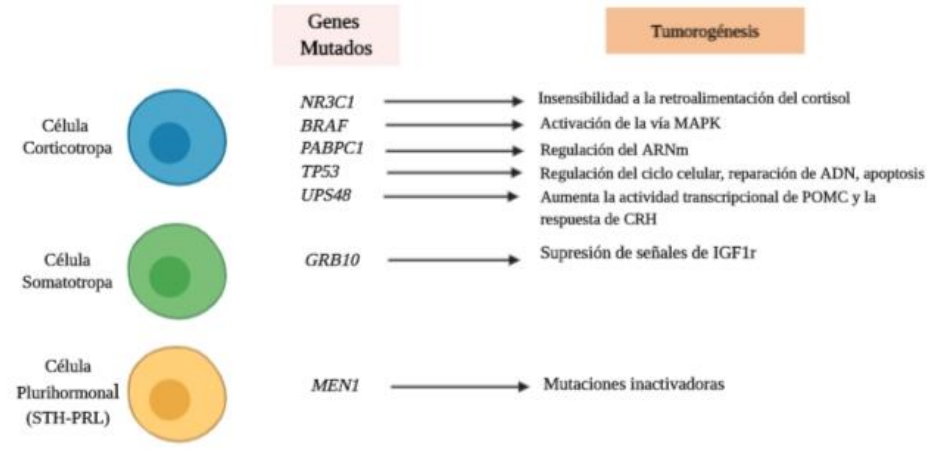

Figura 3: Representación esquemática de nuevas variantes somáticas mutadas sidentificadas a través de estudios de secuenciación del exoma completo (WES) ${ }^{(40-45)}$. Diseño realizado en BioRender.com

\section{B) Cambios epigenéticos}

Si bien la línea germinal y las alteraciones genéticas somáticas han proporcionado información interesante y relevante sobre la tumorogénesis hipofisaria, la mayoría de los tumores hipofisarios son esporádicos y no se conocen mutaciones impulsoras somáticas. Los cambios epigenéticos son modificaciones fenotípicas heredables, que no alteran la secuencia de ADN. Estas pueden regular la transcripción y/o traducción. Los mecanismos epigenéticos pueden ocurrir a nivel de la cromatina, como la metilación del ADN y la modificación de histonas, o a nivel del ARN, mediado principalmente por ARN no codificantes, como microARN, ARN largo no codificante (ARNnc), ARN circular (ARNc circular) entre otros. Dichos mecanismos afectan la expresión génica y, en consecuencia, la tumorogénesis, y han asumido una gran importancia, especialmente dada la escasez de mutaciones somáticas ${ }^{46}$. Se ha descrito la correlación entre modificación epigenética de algunos genes y ciertas características clínicas de los adenomas hipofisarios, como invasividad y tamaño tumoral ${ }^{47}$.

- Metilación del ADN: es uno de los mecanismos epigenéticos más importantes y se ha identificado también en la oncogénesis hipofisaria. Se ha demostrado que los genes CDKN2A, MEG3A, RB, CASP-8, PTAG, FGFR2 y GADD45y presentan modificaciones por cambios en 
la metilación del promotor (hipo o hipermetilación), alterando el ciclo celular $^{48}$. La metilación del ADN está asociada con características clínicas, tales como: subtipo y tamaño tumoral, invasión local, progresión de la enfermedad y respuesta al tratamiento ${ }^{48}$.

- Modificaciones en histonas: la acetilación, la metilación y la citrulinación de histonas están asociadas con regiones activas e inactivas del genoma. Estas marcas epigenéticas pueden ser modificadas por reguladores de cromatina tales como: histona acetiltransferasas, histona desacetilasas, histona metiltransferasas y enzimas de citrulinación. Todos estos mecanismos juegan un papel crucial en la tumorogénesis pituitaria. Por ejemplo: el PTTG es un factor de crecimiento con un papel bien establecido en la patogénesis e invasión hipofisaria y se encuentra sobreexpresado en estos adenomas, como consecuencia de la acetilación de histonas ${ }^{49}$.

- MicroARN: son moléculas pequeñas (aproximadamente 19-25 nucleótidos) que no codifican ARN y que participan en la regulación postranscripcional actuando como activadores o inhibidores de la carcinogénesis $^{48}$. Se los relaciona con el tipo de tumor, las características (tamaño, invasión) y la respuesta al tratamiento. También se ha observado que participan en la regulación de varios genes que se cree que están implicados en la patogénesis de los adenomas hipofisarios ${ }^{50}$.

\section{CONCLUSIÓN}

El conocimiento e información sobre los mecanismos asociados a la formación de tumores hipofisarios incrementó a lo largo de las últimas décadas, y aunque en la actualidad existen pocos enfoques terapéuticos para actuar específicamente sobre la vía de génesis tumoral subyacente, identificada en cada caso, es probable que en el futuro se alcancen avances significativos en este ámbito que aporten beneficios a la hora de decidir el mejor tratamiento para los pacientes. Asimismo, el estudio de la tumorogénesis hipofisaria dentro del contexto de síndromes familiares, no sólo permite la implementación de protocolos de control y vigilancia para los casos índice, sino también, brinda la posibilidad de realizar un diagnóstico y tratamiento oportuno al grupo familiar afectado.

Limitaciones de responsabilidad

La responsabilidad del trabajo es sólo de los autores

Conflictos de interés

Ninguno

Fuentes de apoyo

No posee.

\section{Originalidad del trabajo}

Este artículo es original y no ha sido enviado para su publicación a otro medio de difusión científica en forma completa ni parcialmente.

\section{Cesión de derechos}

Los participantes de este trabajo ceden el derecho de autor a la Universidad Nacional de Córdoba para publicar en la Revista de la Facultad de Ciencias Médicas y realizar las traducciones necesarias al idioma inglés.
Todos los autores hemos participado en la concepción del diseño, recolección de la información y elaboración del manuscrito, haciéndose públicamente responsables de su contenido y aprobando su versión final.

\section{Bibliografía}

1. Melmed S. Mechanisms for pituitary tumorigenesis: the plastic pituitary. J Clin Invest. 2003 Dec;112(11):1603-18. doi: 10.1172/JCI20401.

2. Würth $R$, Thellung $S$, Corsaro A, Barbieri $F$, Florio $T$. Experimental Evidence and Clinical Implications of Pituitary Adenoma Stem Cells. Front Endocrinol (Lausanne). 2020 Feb 20;11:54. doi: 10.3389/fendo.2020.00054.

3. Mantovani G, Giardino E, Treppiedi D, Catalano R, Mangili $F$, Spada A, Arosio M, Peverelli E. Stem Cells in Pituitary Tumors: Experimental Evidence Supporting Their Existence and Their Role in Tumor Clinical Behavior. Front Endocrinol (Lausanne). 2019 Oct 25;10:745. doi: 10.3389/fendo.2019.00745.

4. Ezzat S, Asa SL, Couldwell WT, Barr CE, Dodge WE, Vance ML, McCutcheon IE. The prevalence of pituitary adenomas: a systematic review. Cancer. 2004 Aug 1;101(3):613-9. doi: 10.1002/cncr.20412.

5. Asa SL, Casar-Borota O, Chanson P, Delgrange E, Earls $P$, Ezzat S, Grossman A, Ikeda $H$, Inoshita N, Karavitaki $N$, Korbonits $M$, Laws ER Jr, Lopes MB, Maartens $N$, McCutcheon IE, Mete O, Nishioka H, Raverot G, Roncaroli $F$, Saeger $W$, Syro LV, Vasiljevic $A$, Villa $C$, Wierinckx $A$, Trouillas J; attendees of 14th Meeting of the International Pituitary Pathology Club, Annecy, France, November 2016. From pituitary adenoma to pituitary neuroendocrine tumor (PitNET): an International Pituitary Pathology Club proposal. EndocrRelat Cancer. 2017 Apr;24(4):C5-C8. doi: 10.1530/ERC-17-0004.

6. Osamura RY, Grossman AB, Korbonits M, Kovacs $K$, Lopes MBS, Matsuno A, Trouillas J. Pituitary adenoma. In: Lloyd RV, Osamura RY, Klöppel G, Rosai J, eds. World Health Organization Classification of Tumours of Endocrine Organs. Vol 10. 4th ed. Geneva: International Agency for Cancer Research; 2017:15-19.

7. Zatelli MC. Pathogenesis of non-functioning pituitary adenomas. Pituitary. 2018 Apr;21(2):130-137. doi: 10.1007/s11102-018-0874-6.

8. Malumbres $M$, Barbacid M. Mammalian cyclin-dependent kinases. Trends Biochem Sci. 2005 Nov;30(11):630-41. doi: 10.1016/j.tibs.2005.09.005. Epub 2005 Oct 19.

9. Bilodeau S, Roussel-Gervais A, Drouin J. Distinct developmental roles of cell cycle inhibitors p57Kip2 and p27Kip1 distinguish pituitary progenitor cell cycle exit from cell cycle reentry of differentiated cells. Mol Cell Biol. 2009 Apr;29(7):1895-908. doi: 10.1128/MCB.01885-08. Epub 2009 Jan 12.

10. Tong $Y$, Tan $Y$, Zhou C, Melmed S. Pituitary tumor transforming gene interacts with $S p 1$ to modulate G1/S cell phase transition. Oncogene. 2007 Aug 16;26(38):5596-605. doi: 10.1038/sj.onc. 1210339.

11. Park MS, Rosai J, Nguyen HT, Capodieci P, Cordon-Cardo C, Koff A. p27 and Rb are on overlapping pathways suppressing tumorigenesis in mice. Proc Natl Acad Sci U S A. 1999 May 25;96(11):6382-7. doi: 10.1073/pnas.96.11.6382.

12. Ramsey MR, Krishnamurthy J, Pei XH, Torrice C, Lin W, Carrasco DR, Ligon KL, Xiong Y, Sharpless NE. Expression of p16Ink4a compensates for p18Ink4c loss in cyclindependent kinase 4/6-dependent tumors and tissues. Cancer 
13. Res. 2007 May 15;67(10):4732-41. doi: 10.1158/00085472. CAN-06-3437.

14. Roussel-Gervais A, Bilodeau S, Vallette $S$, et al. Cooperation between cyclin $E$ and p27(Kip1) in pituitary tumorigenesis. Mol Endocrinol. 2010;24(9):1835-1845. doi:10.1210/me.2010-0091.

15. Franklin DS, Godfrey VL, O'Brien DA, Deng C, Xiong Y. Functional collaboration between different cyclin-dependent kinase inhibitors suppresses tumor growth with distinct tissue specificity. Mol Cell Biol. 2000 Aug;20(16):6147-58. doi: 10.1128/mcb.20.16.6147-6158.2000.

16. Sotillo $R$, Renner $O$, Dubus $P$, Ruiz-Cabello J, MartínCaballero J, Barbacid $M$, Carnero A, Malumbres $M$. Cooperation between Cdk4 and p27kip1 in tumor development: a preclinical model to evaluate cell cycle inhibitors with therapeutic activity. Cancer Res. 2005 May 1;65(9):3846-52. doi: 10.1158/0008-5472.CAN-04-4195.

17. Agarwal SK, Mateo CM, Marx SJ. Rare germline mutations in cyclin-dependent kinase inhibitor genes in multiple endocrine neoplasia type 1 and related states. J Clin Endocrinol Metab. 2009 May;94(5):1826-34. doi: 10.1210/jc.2008-2083. Epub 2009 Jan 13. Erratum in: J Clin Endocrinol Metab. 2009 Jul;94(7):2674.

18. Hernández-Ramírez LC, Gabrovska $P$, Dénes J, Stals K, Trivellin G, Tilley D, Ferrau F, Evanson J, Ellard S, Grossman $A B$, Roncaroli $F$, Gadelha MR, Korbonits M; International FIPA Consortium. Landscape of Familial Isolated and YoungOnset Pituitary Adenomas: Prospective Diagnosis in AIP Mutation Carriers. J Clin Endocrinol Metab. 2015 Sep;100(9):E1242-54. doi: 10.1210/jc.2015-1869.

19. Daly AF, Tichomirowa MA, Petrossians $P$, Heliövaara $E$, Jaffrain-Rea ML, Barlier A, Naves $L A$, Ebeling $T$, Karhu A, Raappana A, Cazabat L, De Menis E, Montañana CF, Raverot G, Weil RJ, Sane T, Maiter D, Neggers S, Yaneva M, Tabarin A, Verrua E, Eloranta E, Murat A, Vierimaa $O$, Salmela PI, Emy $P$, Toledo RA, Sabaté MI, Villa C, Popelier $M$, Salvatori R, Jennings J, Longás AF, LabartaAizpún Jl, Georgitsi M, Paschke R, Ronchi C, Valimaki M, Saloranta C, De Herder W, Cozzi R, Guitelman M, Magri F, Lagonigro MS, Halaby G, Corman V, Hagelstein MT, Vanbellinghen JF, Barra GB, Gimenez-Roqueplo AP, Cameron FJ, BorsonChazot F, Holdaway I, Toledo SP, Stalla GK, Spada A, Zacharieva S, Bertherat J, Brue $T$, Bours V, Chanson $P$, Aaltonen $L A$, Beckers A. Clinical characteristics and therapeutic responses in patients with germ-line AIP mutations and pituitary adenomas: an international collaborative study. J Clin Endocrinol Metab. 2010 Nov;95(11):E373-83. doi: 10.1210/jc.2009-2556

20. Oriola J, Lucas T, Halperin I, Mora M, Perales MJ, AlvarezEscolá C, Paz de MN, Díaz Soto G, Salinas I, Julián MT, Olaizola I, Bernabeu I, Marazuela M, Puig-Domingo M. Germline mutations of AIP gene in somatotropinomas resistant to somatostatin analogues. Eur J Endocrinol. 2012 Dec 10;168(1):9-13. doi: 10.1530/EJE-12-0457.

21. Korbonits M, Storr H, Kumar AV. Familial pituitary adenomas - who should be tested for AIP mutations? Clin Endocrinol (Oxf). 2012 Sep;77(3):351-6. doi: 10.1111/j.13652265.2012.04445.x.

22. Beckers A, Lodish MB, Trivellin G, Rostomyan L, Lee $M$, Faucz FR, Yuan B, Choong CS, Caberg JH, Verrua E, Naves LA, Cheetham TD, Young J, Lysy PA, Petrossians P, Cotterill A, Shah NS, Metzger D, Castermans E, Ambrosio MR, Villa C, Strebkova N, Mazerkina N, Gaillard S, Barra GB, Casulari $L A$, Neggers SJ, Salvatori R, Jaffrain-Rea ML, Zacharin $M$, Santamaria BL, Zacharieva S, Lim EM, Mantovani G, Zatelli MC, Collins MT, Bonneville JF, Quezado M, Chittiboina $P$, Oldfield EH, Bours V, Liu P, W de Herder W, Pellegata N,
Lupski JR, Daly AF, Stratakis CA. X-linked acrogigantism syndrome: clinical profile and therapeutic responses. EndocrRelat Cancer. 2015 Jun;22(3):353-67. doi: 10.1530/ERC-15-0038. Epub 2015 Feb 24.

23. lacovazzo $D$, Caswell $R$, Bunce $B$, Jose $S$, Yuan $B$, Hernández-Ramírez LC, Kapur S, Caimari F, Evanson J, Ferraù F, Dang MN, Gabrovska P, Larkin SJ, Ansorge O, Rodd $C$, Vance ML, Ramírez-Renteria $C$, Mercado $M$, Goldstone AP, Buchfelder M, Burren CP, Gurlek A, Dutta $P$, Choong CS, Cheetham T, Trivellin G, Stratakis CA, Lopes MB, Grossman AB, Trouillas J, Lupski JR, Ellard S, Sampson $J R$, Roncaroli F, Korbonits M. Germline or somatic GPR101 duplication leads to $X$-linked acrogigantism: a clinicopathological and genetic study. Acta NeuropatholCommun. 2016 Jun 1;4(1):56. doi: 10.1186/s40478-016-0328-1.

24. Hernández-Ramírez LC, Gam R, Valdés $N$, Lodish MB, Pankratz N, Balsalobre A, Gauthier Y, Faucz FR, Trivellin G, Chittiboina P, Lane J, Kay DM, Dimopoulos A, Gaillard S, Neou M, Bertherat J, Assié G, Villa C, Mills JL, Drouin J, Stratakis CA. Loss-of-function mutations in the CABLES1 gene are a novel cause of Cushing's disease. EndocrRelat Cancer. 2017 Aug;24(8):379-392. doi: 10.1530/ERC-170131.

25. Bernard V, Bouilly J, Beau I, Broutin I, Chanson P, Young J, Binart N. Germline Prolactin Receptor Mutation Is Not a Major Cause of Sporadic Prolactinoma in Humans. Neuroendocrinology. 2016;103(6):738-45. doi: $10.1159 / 000442981$

26. Melo FM, Couto PP, Bale AE, Bastos-Rodrigues L, Passos FM, Lisboa RG, Ng JM, Curran T, Dias EP, Friedman E, De Marco L. Whole-exome identifies RXRG and TH germline variants in familial isolated prolactinoma. Cancer Genet. 2016 Jun;209(6):251-7. doi: 10.1016/j.cancergen.2016.05.065.

27. Rostad S. Pituitary adenoma pathogenesis: anupdate. CurrOpinEndocrinol Diabetes Obes. 2012 Aug;19(4):322-7. doi: 10.1097/MED.0b013e328354b2e2.

28. Pellegata NS, Quintanilla-Martinez L, Siggelkow H, Samson E, Bink K, Höfler H, Fend F, Graw J, Atkinson MJ. Germ-line mutations in p27Kip1 cause a multiple endocrine neoplasia syndrome in rats and humans. Proc Natl Acad Sci U S A. 2006 Oct 17;103(42):15558-63. doi: 10.1073/pnas.0603877103. Epub 2006 Oct 9. Erratum in: Proc Natl Acad Sci U S A. 2006 Dec 12;103(50):19213.

29. Bertherat J, Horvath A, Groussin L, Grabar S, Boikos S, Cazabat L, Libe R, René-Corail F, Stergiopoulos S, Bourdeau I, Bei T, Clauser E, Calender A, Kirschner LS, Bertagna X, Carney JA, Stratakis $C A$. Mutations in regulatory subunit type $1 A$ of cyclic adenosine 5'-monophosphate-dependent protein kinase (PRKAR1A): phenotype analysis in 353 patients and 80 different genotypes. J Clin Endocrinol Metab. 2009 Jun;94(6):2085-91. doi: 10.1210/jc.2008-2333.

30. de Kock L, Sabbaghian N, Plourde F, Srivastava A, Weber E, Bouron-Dal Soglio D, Hamel N, Choi JH, Park SH, Deal CL, Kelsey MM, Dishop MK, Esbenshade A, Kuttesch JF, Jacques TS, Perry A, Leichter H, Maeder P, Brundler MA, Warner J, Neal J, Zacharin $M$, Korbonits $M$, Cole $T$, Traunecker H, McLean TW, Rotondo F, Lepage P, Albrecht $S$, Horvath E, Kovacs K, Priest JR, Foulkes WD. Pituitary blastoma: a pathognomonic feature of germ-line DICER1 mutations. Acta Neuropathol. 2014 Jul;128(1):111-22. doi: 10.1007/s00401-014-1285-z.

31. Xekouki $P$, Szarek E, Bullova P, Giubellino A, Quezado M, Mastroyannis SA, Mastorakos $P$, Wassif CA, Raygada M, Rentia N, Dye L, Cougnoux A, Koziol D, Sierra Mde L, Lyssikatos C, Belyavskaya E, Malchoff C, Moline J, Eng C, Maher LJ 3rd, Pacak K, Lodish M, Stratakis CA. Pituitary adenoma with paraganglioma/pheochromocytoma (3PAs) 
and succinate dehydrogenase defects in humans and mice. $J$ Clin Endocrinol Metab. 2015 May;100(5):E710-9. doi: 10.1210/jc.2014-4297. Epub 2015 Feb 19.

32. Uraki S, Ariyasu H, Doi A, Furuta $H$, Nishi M, Sugano K, Inoshita N, Nakao N, Yamada S, Akamizu T. Atypical pituitary adenoma with MEN1 somatic mutation associated with abnormalities of DNA mismatch repair genes; $M L H 1$ germline mutation and MSH6 somatic mutation. Endocr J. 2017 Sep 30;64(9):895-906. doi: 10.1507/endocrj.EJ17-0036.

33. Cohen M, Persky R, Stegemann R, Hernández-Ramírez LC, Zeltser D, Lodish MB, Chen A, Keil MF, Tatsi C, Faucz FR, Buchner DA, Stratakis CA, Tiosano D. Germline USP8 Mutation Associated With Pediatric Cushing Disease and Other Clinical Features: A New Syndrome. J Clin Endocrinol Metab. 2019 Oct 1;104(10):4676-4682. doi: 10.1210/jc.201900697.

34. Tigas S, Carroll PV, Jones R, Bingham E, Russell-Jones D, Powell M, Scobie IN. Simultaneous Cushing's disease and tuberous sclerosis; a potential role for TSC in pituitary ontogeny. Clin Endocrinol (Oxf). 2005 Dec;63(6):694-5. doi: 10.1111/j.1365-2265.2005.02374.x.

35. Välimäki N, Demir H, Pitkänen E, Kaasinen E, Karppinen A, Kivipelto L, Schalin-Jäntti $C$, Aaltonen LA, Karhu A. WholeGenome Sequencing of Growth Hormone (GH)-Secreting Pituitary Adenomas. J Clin Endocrinol Metab. 2015 Oct;100(10):3918-27. doi: 10.1210/jc.2015-3129

36. Song ZJ, Reitman ZJ, Ma ZY, Chen JH, Zhang QL, Shou XF, Huang CX, Wang YF, Li SQ, Mao Y, Zhou LF, Lian BF, Yan $H$, Shi $Y Y$, Zhao $Y$. The genome-wide mutational landscape of pituitary adenomas. Cell Res. 2016 Nov;26(11):1255-1259. doi: 10.1038/cr.2016.114. Epub 2016 Sep 27.

37. Freda PU, Chung WK, Matsuoka N, Walsh JE, Kanibir MN, Kleinman G, Wang Y, Bruce JN, Post KD. Analysis of GNAS mutations in 60 growth hormone secreting pituitary tumors: correlation with clinical and pathological characteristics and surgical outcome based on highly sensitive GH and IGF-I criteria for remission. Pituitary. 2007;10(3):275-82. doi: 10.1007/s11102-007-0058-2.

38. Neou M, Villa $C$, Armignacco $R$, Jouinot A, Raffin-Sanson ML, Septier A, Letourneur F, Diry S, Diedisheim M, Izac B, Gaspar $C$, Perlemoine $K$, Verjus $V$, Bernier $M$, Boulin A, Emile JF, Bertagna $X$, Jaffrezic $F$, Laloe $D$, Baussart B, Bertherat $J$, Gaillard S, Assié G. Pangenomic Classification of Pituitary Neuroendocrine Tumors. Cancer Cell. 2020 Jan 13;37(1):123-134.e5. doi: 10.1016/j.ccell.2019.11.002.

39. Albright F, Butler AM, Hampton AO, Smith P. Syndrome Characterized by Osteitis Fibrosa Disseminata, Areas of Pigmentation and Endocrine Dysfunction, with Precocious Puberty in Females. N Engl J Med 1937; 216:727-746.

40. Reincke $M$, Sbiera S, Hayakawa A, Theodoropoulou M, Osswald A, Beuschlein F, Meitinger T, Mizuno-Yamasaki E, Kawaguchi K, Saeki Y, Tanaka K, Wieland T, Graf E, Saeger W, Ronchi CL, Allolio B, Buchfelder M, Strom TM, Fassnacht $M$, Komada $M$. Mutations in the deubiquitinase gene USP8 cause Cushing's disease. Nat Genet. 2015 Jan;47(1):31-8. doi: 10.1038/ng.3166.

41. Kurelac I, MacKay A, Lambros MB, Di Cesare E, Cenacchi G, Ceccarelli C, Morra I, Melcarne A, Morandi L, Calabrese FM, Attimonelli M, Tallini G, Reis-Filho JS, Gasparre G. Somatic complex I disruptive mitochondrial DNA mutations are modifiers of tumorigenesis that correlate with low genomic instability in pituitary adenomas. Hum Mol Genet. 2013 Jan 15;22(2):226-38. doi: 10.1093/hmg/dds422.

42. Bruns C, Lewis I, Briner U, Meno-Tetang G, Weckbecker G. SOM230: a novel somatostatin peptidomimetic with broad somatotropin release inhibiting factor (SRIF) receptor binding and a unique antisecretory profile. Eur J Endocrinol. 2002 May;146(5):707-16. doi: 10.1530/eje.0.1460707.

43. Perez-Rivas LG, Theodoropoulou M, Ferraù F, Nusser C, Kawaguchi K, Stratakis CA, Faucz FR, Wildemberg LE, Assié G, Beschorner R, Dimopoulou C, Buchfelder M, Popovic V, Berr CM, Tóth M, Ardisasmita Al, Honegger J, Bertherat J, Gadelha MR, Beuschlein F, Stalla G, Komada M, Korbonits $M$, Reincke M. The Gene of the Ubiquitin-Specific Protease 8 Is Frequently Mutated in Adenomas Causing Cushing's Disease. J Clin Endocrinol Metab. 2015 Jul;100(7):E9971004. doi: 10.1210/jc.2015-1453. Epub 2015 May 5.

44. Losa M, Mortini P, Pagnano A, Detomas M, Cassarino MF, PecoriGiraldi F. Clinical characteristics and surgical outcome in USP8-mutated human adrenocorticotropic hormonesecreting pituitary adenomas. Endocrine. 2019 Feb;63(2):240-246. doi: 10.1007/s12020-018-1776-0.

45. Bengtsson $D$, Joost $P$, Aravidis $C$, AskmalmStenmark $M$, Backman AS, Melin B, von Salomé J, Zagoras T, GebreMedhin S, Burman P. Corticotroph Pituitary Carcinoma in a Patient With Lynch Syndrome (LS) and Pituitary Tumors in a Nationwide LS Cohort. J Clin Endocrinol Metab. 2017 Nov 1;102(11):3928-3932. doi: 10.1210/jc.2017-01401.

46. Tanizaki $Y$, Jin L, Scheithauer BW, Kovacs $K$, Roncaroli $F$, Lloyd RV. P53 gene mutations in pituitary carcinomas. EndocrPathol. 2007 Winter;18(4):217-22. doi: 10.1007/s12022-007-9006-y.

47. SrirangamNadhamuni $V$, Korbonits $M$. Novel insights into Pituitary Tumorigenesis: Genetic and Epigenetic Mechanisms. Endocr Rev. 2020 Mar 23:bnaa006. doi: 10.1210/endrev/bnaa006. Epub ahead of print.

48. Pease M, Ling C, Mack WJ, Wang K, Zada G. The role of epigenetic modification in tumorigenesis and progression of pituitary adenomas: a systematic review of the literature. PLoS One. 2013 Dec 18;8(12):e82619. doi: 10.1371/journal.pone.0082619. PMID: 24367530;

49. Dudley KJ, Revill K, Clayton RN, Farrell WE. Pituitary tumours: all silent on the epigenetics front. $J \mathrm{Mol}$ Endocrinol. 2009 Jun;42(6):461-8. doi: 10.1677/JME-09-0009

50. García-Martínez A, Sottile J, Sánchez-Tejada L, Fajardo C, Cámara R, Lamas C, Barberá VM, Picó A. DNA Methylation of Tumor Suppressor Genes in Pituitary Neuroendocrine Tumors. J Clin Endocrinol Metab. 2019 Apr 1;104(4):12721282. doi: 10.1210/jc.2018-01856.

51. Gadelha MR, Kasuki L, Dénes J, Trivellin G, Korbonits M. MicroRNAs: Suggested role in pituitary adenoma pathogenesis. J Endocrinol Invest. 2013 Nov;36(10):889-95. doi: $10.1007 / B F 03346759$. 